\title{
Evaluer les activités en faveur de la qualité afin de faciliter les choix
}

\begin{abstract}
Les différentes disciplines médicales proposent des activités très diverses pour optimiser les processus de travail et réduire les erreurs. Or, nombre de ces activités signifient une charge de travail et des coûts plus importants pour les médecins.

- Il est nécessaire de procéder à une analyse approfondie des différentes activités en faveur de la qualité selon leur rapport coûts-utilité et de prendre en compte de manière appropriée les différents aspects liés aux coûts ou à l'utilité.

- La FMH assume principalement un rôle de coordination lorsqu'il s'agit de définir des activités appropriées en faveur de la qualité. Elle soutient également l'élaboration d'une grille d'évaluation adéquate. Mais au final, ce sont les sociétés de discipline médicale qui décident quelles démarches qualité elles souhaitent recommander.

- Il s'agit de faire connaître les démarches qualité judicieuses, afin que les médecins soient mieux informés dans leur choix de mesures appropriées.
\end{abstract}

\section{Contexte initial}

Les activités visant à promouvoir et à assurer la qualité sont une évidence pour la majorité des médecins suisses. La promotion de la qualité est en outre ancrée dans le code de déontologie de la FMH. La liste des activités en faveur de la qualité est longue et elle varie selon les disciplines médicales. Etant donné que les ressources sont limitées et que toutes les démarches qualité ne peuvent être accomplies dans la pratique, un choix s'impose pour les médecins, et le rapport coûts-utilité joue ici un rôle primordial. Aujourd'hui, nous ne disposons toutefois d'aucune théorie prépondérante pour évaluer les activités en faveur de la qualité. Il n'existe aucune méthode d'évaluation pratique du rapport coûts-utilité.

\section{La position de la FMH}

Coûts et utilité des activités en faveur de la qualité Toute activité en faveur de la qualité a son prix. La mise en œuvre de ces démarches signifie fréquemment une importante charge supplémentaire pour les médecins, car celles-ci nécessitent non seulement du temps, mais engendrent aussi des coûts matériels et de personnel ainsi que des frais pour le recours à des prestations de tiers. Mais les activités en faveur de la qualité peuvent aussi être d'une grande utilité. Si elles sont judicieuses et pratiques, elles permettent d'optimiser les processus de travail et de réduire les erreurs, ce qui a un impact positif sur la sécurité des patients et la qualité des traitements. Simultanément, cette démarche permet d'accroître la satisfaction des patients mais aussi celle des médecins traitants.

\section{Evaluation des activités en faveur de la qualité}

Chaque activité en faveur de la qualité bénéficie de son propre rapport coûts-utilité. Etant donné que seules les activités dont l'utilité justifie les coûts entrent en ligne de compte, il est judicieux d'évaluer ces deux aspects, et ce d'autant plus que les ressources sont limitées dans le domaine de la santé et que, sur le plan des coûts, ces démarches qualité entrent en concurrence avec les autres dépenses. Or, pour le médecin, il importe de savoir quelles activités peuvent, moyennant un coût raisonnable, s'avérer d'une grande utilité.

\section{Définition des activités qualité judicieuses}

Actuellement, de nombreuses démarches qualité sont déployées sur plusieurs disciplines, la collaboration est donc de mise. A cet effet, la FMH souhaite assumer principalement un rôle de coordination afin d'éviter les doublons. Elle soutient également l'élaboration d'une grille d'évaluation adéquate. Et ce sera finalement aux sociétés de discipline médicale de définir quelles activités en faveur de la qualité sont judicieuses. Elles sont en effet les seules à même de réaliser une analyse spécifique du rapport coûtsutilité dans leur discipline et d'informer leurs membres quant au choix des démarches qualité. Leur collaboration est donc primordiale lors de l'élaboration d'une grille d'évaluation.

\section{Communication et promotion de démarches qualité appropriées}

Les activités en faveur de la qualité qui s'avèrent judicieuses doivent être communiquées à l'interne par les sociétés de discipline médicale. Ceci dans le but d'aider les médecins à choisir quelles mesures de promotion et de développement de la qualité adopter. De plus, il s'agira de les promouvoir par des incitatifs appropriés. Là aussi, les mesures d'accompagnement et l'indemnisation des charges administratives et financières supplémentaires sont primordiales pour les médecins. La FMH élabore actuellement des solutions ciblées dans ce sens. 